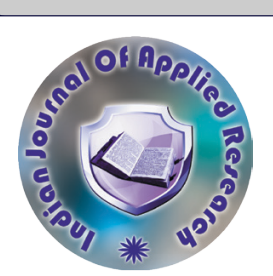

Microbiology

\title{
KNOWLEDGE, ATTITUDE AND PRACTICES OF HAND HYGIENE IN A HEALTH-CARE SETTING
}

Dr. A. Seetha

M.D, Assistant Professor, Department Of Microbiology, Government Sivagangai Medical College, Sivagangai

ABSTRACT

AIM: explore the existing knowledge, attitudes and practices with regard to Hand hygine (HH), the total $\mathrm{HH}$ compliance and the various barriers to $\mathrm{HH}$ in our hospital so as to plan the strategies for bridging these gaps, thus improving patient care. METHODS AND MATERIALS A cross-sectional study was conducted among $300 \mathrm{HCWs}$ of Madurai Medical College, Madurai belonging to six different categories, for a period of 2 months. Knowledge, attitude and practices were evaluated using a validated WHO HH questionnaire, a self-structured attitude questionnaire and an observation checklist based on the concept of Five Moments of HH by WHO, respectively. Total compliance and the profile of missed opportunities were also assessed. SPSS was used for data analysis. RESULTS: The knowledge on $\mathrm{HH}$ was good (190 out of 300, 63.3\%), attitudes were poor with nursing staff having significantly better attitude than doctors (P $<$ 0.05). In the observational study, physicians and nursing staff had better practice. Lack of time was the major barrier pointed out. The overall compliance was $46 \%$ among the HCWs of whom only $16 \%$ had proper $\mathrm{HH}$ practice. Rest of the $30 \%$ performed $\mathrm{HH}$, but the technique was wrong. CONCLUSIONS: The study highlights the need for reinforcing the existing $\mathrm{HH}$ training programme to address the gaps in knowledge, attitude and practice and thereby improving the level of $\mathrm{HH}$ compliance and enhancing patient safety.

KEYWORDS : Hand hygine, knowledge, attitude, hand washing

\section{INTRODUCTION}

Patients receiving health care, are at risk of HAI ranging from $6 \%$ to $27 \%$ in the developing and resource-poor settings.[1]The prevalence of HAIs due to poor hand hygiene $(\mathrm{HH})$ is about $19 \%$ in developing countries.[2] Basically, HH depends on the attitude, behaviour and beliefs of an individual.[3] Hence, this study strives to focus on the knowledge, attitude and practices, which are the three main determinants of $\mathrm{HH}$ in a tertiary health-care set up. By measuring the $\mathrm{HH}$ compliance and identifying the barriers, effective protocols for infection prevention can be designed, implemented and taught to improve health-care outcomes in the future.

\section{AIM}

To assess the knowledge, attitude and practice of $\mathrm{HH}$ in our hospital among the various categories of health-care workers.

The objectives of the study include:

1. Assess the knowledge of and attitude towards $\mathrm{HH}$ in senior and junior doctors, medical students, staff nurses, nursing students and housekeeping staff

Assess the compliance and to find out the profile of missed opportunities in $\mathrm{HH}$.

\section{METHODS\&MATERIALS:}

This is a descriptive type of study with cross-sectional study design conducted by the Infection Control team under the institute of microbiology Madurai medical college, Madurai, India for 2 month.According to a study done by Pittet et al., the average prevalence of $\mathrm{HH}$ was found to be $40 \%$ and hence using the formula $4 \mathrm{pq} / \mathrm{l}^{2}$ a sample size of 300 was arrived The study group consisted of senior and junior doctors, medical students, staff nurses, nursing students and housekeeping staff, with 50 samples from each category. As it was a short term and preliminary study to get an overall idea on the existing $\mathrm{HH}$ practise in our hospital, further categorisation of study groups such as intensive care units and high dependency units which may reflect a change in their priority towards $\mathrm{HH}$ was not done. In this three-tier study, the knowledge and attitude were assessed using the WHO's HH questionnaire for $\mathrm{HCWs}$ [5] and a self-structured attitude questionnaire adapted from previous studies, respectively[6]. Housekeeping staff were given a questionnaire in their language, omitting some of the questions which were not applicable to them. The responses were evaluated and knowledge was categorised as good $(\geq 75 \%)$, moderate $(50 \%-75 \%)$ and poor $(\leq 50 \%)$ of the total score. The attitude statements were evaluated on the basis of a scale of $0-4$, with 0 as "don't know" and 4 as "strongly agree." [7] Statistical analysis of the data was carried out using the Chi-square test.

\section{RESULTS}

A total of $300 \mathrm{HCWs}$ were evaluated on their knowledge, attitude and practise of $\mathrm{HH}$ which consisted of senior and junior doctors, medical students, staff nurses, nursing students and housekeeping staff, with 50 samples from each category and this sample size was obtained using the formula $4 \mathrm{pq} / 1^{2}$. Since this was a short-term and preliminary study to get an overall idea on the existing $\mathrm{HH}$ practise in our hospital, further categorisation of study groups such as intensive care units and high dependency units., which may reflect a change in their priority towards $\mathrm{HH}$ were not doneOut of 300 participants, $190(63.3 \%)$ had a total knowledge score of more than $75 \%$ which pertains to the whole study group from all 6 categories. Thirty-seven (74\%) of both senior and junior doctors had good scores (above $75 \%$ ), whereas $33(66 \%$ ) of both medical and nursing students, $28(56 \%)$ of staff nurses and $22(44 \%)$ of housekeeping staff also got good score. None of the HCWs had poor scores $(<50 \%)$ except one house keeping staff $(2 \%)$. The scores obtained for each question by each category of HCW is Only $19(38 \%)$ of senior doctors, $26(52 \%)$ of junior doctors and $7(14 \%)$ of medical students have received proper formal training in $\mathrm{HH}$ in the past 3 years. About $44(88 \%)$ of the staff nurses and $37(74 \%)$ of the senior physicians preferred to use the alcohol based hand rub routinely instead of hand washing with soap and water. While looking for the important gaps in the clinical knowledge based on the responses to the individual questions we found that only 29 (58\%) senior doctors, 22 (44\%) junior doctors, 24 (48\%) medical students, 19 (38\%) staff nurses and 17 (34\%) nursing students knew the minimal time $(20 \mathrm{~s})$ which was needed for an alcohol based hand rub to kill most germs on the hand.While assessing the attitudes among the HCWs, nurses seemed to have significantly better attitudes when compared to rest of the study population $(P<0.05)$. Respondents found that emergencies and other priorities often make it difficult for them to adhere to $\mathrm{HH}$ at all times and they often feel frustrated when they omit HH. As per the scoring system, both these statements scored an average above 3.5 . Overall $78 \%(236 / 300)$ and $83.33 \%(250 / 300)$ of HCWs commented that they strongly agree (score 4 ) that infection prevention team has a positive influence on their $\mathrm{HH}$ and infection prevention notice boards remind them to perform $\mathrm{HH}$

\section{DISCUSSION}

The medical community all over the world witnessed a tandem unprecedented advancement in the understanding of pathophysiology of Hospital Acquired Infectious (HAI) diseases due to its increasing influence on the magnitude of problems such as morbidity, mortality and hospital economy. Compliance with HH guidelines has been considered to be the most important strategy to reduce the transmission of HAIs in health-care settings for many years.[4] In spite of the overwhelming evidence demonstrating the negative consequences of HAIs and on-going education emphasising the importance of performing $\mathrm{HH}$, low $\mathrm{HH}$ compliance rates among the $\mathrm{HCW}$ s continue to prevail.In our study, knowledge of $\mathrm{HH}$ seemed to be good with $63.3 \%$ (190 HCWs) having more than $75 \%$ score when compared to a similar Indian study where in $74 \%$ of the study population, the knowledge was between $50 \%$ and $75 \%$ of total score.[5] Majority of the study population still calls on the need for formal training in $\mathrm{HH}$ 
which was strongly evident in their response to the question of whether they had received any formal training in $\mathrm{HH}$ in the last 3 years. Only $38 \%$ of senior doctors, $52 \%$ of junior doctors and $14 \%$ of medical students had received proper formal training in $\mathrm{HH}$ in the past 3 years. Forty-four $(88 \%)$ of the staff nurses and $37(74 \%)$ of the senior physicians preferred to use the alcohol-based hand rub routinely instead of hand washing with soap and water. This practice of using alcohol based hand rub has been recommended strongly by the WHO in the past few years, as it is a less cumbersome procedure with more residual effect and very useful in a hospital setting where uninterrupted water availability is a question. However, there were some gaps in the clinical knowledge, of which an important finding was that only a minority of the population knew the minimal time $(20 \mathrm{~s})$ needed for an alcohol-based hand rub to kill most germs on the hand. It was known to only $29(58 \%)$ of senior doctors, 22 (44\%) of junior doctors, $24(48 \%)$ of medical students, $19(38 \%)$ of staff nurses and 17 (34\%) of nursing students. While assessing the attitude statements, overall results were disappointing. Our findings suggest that nursing students and staff nurses have significantly better attitudes $(P<0.05) 78 \%$ and $84 \%$ when compared to all the other categories of $\mathrm{HCWs}$, which was found to be similar with the reports from Cairo $(96.0 \%)$ and Italy $(86.2 \%)$. Lack of compliance due to forgetfulness can be countered by placing reminders such as posters, installing wash scans or digital screening of hands, through $\mathrm{HH}$ auditing with regular feedbacks and also by ongoing education processes. Among all $\mathrm{HCWs}$, $78 \%$ commented that infection prevention team has a positive influence on their $\mathrm{HH}$ and $83.33 \%$ of HCWs said that infection prevention notice boards remind them to perform HH. Lack of time (54\%) has been highlighted as the major barrier for adequate performance of $\mathrm{HH}$, especially by the nursing community in our study as was shown in a previous study by Barret and Randle also. in spite of the discrepancies in the high levels of knowledge and poor levels of attitudes and compliance, the nursing community is far ahead of the physicians. This may be due to the longer time of interaction with the patients during their nursing procedures. Furthermore, infection control lessons are being taught in their nursing curriculum itself along with better exposure to proper $\mathrm{HH}$ techniques repeatedly right from a very early stage. Hence, we would like to highlight the importance of improving the current training programmes targeting $\mathrm{HH}$ practices among medical and nursing students and the commonly missed out category of housekeeping staff. Teaching of elementary HH practices along with coupling of lectures in the undergraduate curriculum can be done so as to prime the medical students to this basic necessity of performing HH. Mentor's attitude at bed side which has a strong influence in moulding the behaviour of young medical and nursing students should be exploited to serve as role models for them. Provision of adequate staff and facilities for hand washing with easy access should also be guaranteed.

\section{CONCLUSION}

$\mathrm{HCW}$ are being asked to return to the basics of infection prevention by adhering to simple measures like HH. The present study identified good rates of HH knowledge; while attitudes and practices of $\mathrm{HH}$ were found to be unsatisfactory on the whole. Hence, we highlight the urgent need for introducing and upgrading the existing measures to improve the knowledge, attitude and practise of $\mathrm{HH}$ among the HCWs. Moreover, institutional support for providing necessary incentives for adhering to $\mathrm{HH}$ should also be guaranteed in every hospital setting.

\section{REFERENCES :}

1. Danchaivijitr S, Dhiraputra C, Santiprasitkul S, Judaeng T. Prevalence and impacts of nosocomial infections in Thailand. J Med Assoc Thai 2001;88:S1-9.

2. Ariyaratne MH, Gunasekara TD, Weerasekara MM, Kottahachchi J, Kudavidanage BP, Ariyaratne MH, Gunasekara TD, Weerasekara MM, Kottahachchi J, Kudavidanage BP,
Fernando SS. Knowledge, attitude and practices of hand hygiene among final year Fernando SS. Knowledge, attitude and practices of hand hygiene among final year
medical and nursing students at the University of Sri Jayewardenepura. Sri Lankan J Infect Dis 2013;3;15-25.

3. Pittet D, Mourouga P, Perneger TV. Compliance with handwashing in a teaching hospital. Infection control program. Ann Intern Med 1999;130:126-30.

4. WHO Hand Hygiene Knowledge Questionnaire for Health-Care Workers (Revised August 2009). To Assess Knowledge on the Essential Aspects of HH.

5. Shinde MB, Mohite VR. A study to assess knowledge, attitude and practices of five moments of hand hygiene among nursing staff and students at a tertiary care hospital, Karad. Int J Sci Res 2014;3:311-21.

6. van Dalen R, Gombert K, Bhattacharya S, Datta SS. Mind the mind: Results of a handhygiene research in a state-of-the-art cancer hospital. Indian J Med Microbiol 2013;31:280-2. 